\title{
Dual-Regge approach to high-energy, low-mass diffraction dissociation
}

\author{
L. L. Jenkovszky, ${ }^{1}$ O. E. Kuprash, ${ }^{2}$ J. W. Lämsä, ${ }^{3}$ V. K. Magas, ${ }^{4}$ and R. Orava ${ }^{5,6}$ \\ ${ }^{1}$ Bogolyubov Institute for Theoretical Physics (BITP), Ukrainian National Academy of Sciences, \\ 14-b, Metrolohichna str., Kiev, 03680, Ukraine \\ ${ }^{2}$ Taras Shevchenko National University, Kiev, Ukraine \\ ${ }^{3}$ Physics Department, Iowa State University, Ames, 50011 Iowa, USA \\ ${ }^{4}$ Departament d'Estructura i Constituents de la Matéria, Universitat de Barcelona, Diagonal 647, 08028 Barcelona, Spain \\ ${ }^{5}$ Helsinki Institute of Physics, Division of Elementary Particle Physics, P.O. Box 64 (Gustaf Hällströminkatu 2a), \\ FI-00014 University of Helsinki, Finland \\ ${ }^{6} \mathrm{CERN}, \mathrm{CH}-1211$ Geneva 23, Switzerland \\ (Received 2 November 2010; revised manuscript received 9 February 2011; published 25 March 2011)
}

A dual-Regge model with a nonlinear proton Regge trajectory in the missing mass $\left(M_{X}^{2}\right)$ channel, describing the experimental data on low-mass single diffraction dissociation (SDD), is constructed. Predictions for the LHC energies are given.

DOI: 10.1103/PhysRevD.83.056014

PACS numbers: 11.55. $-\mathrm{m}, 11.55 . \mathrm{Jy}, 12.40 . \mathrm{Nn}$

\section{INTRODUCTION}

The possible existence of a new class of processes, later named diffraction dissociation, for the first time was indicated in 1953, in a short paper by Pomeranchuk and Feinberg [1]. The possibility of observing diffractive inelastic processes producing states $X$ of large mass was studied subsequently, in 1960 by Good and Walker [2] (for a review, see Ref. [3]).

Experimentally, diffraction dissociation in protonproton scattering was intensively studied in the ' 70 -ies at the Fermilab and the CERN ISR [4-6]. In particular, in Ref. [6] double-differential cross section $\frac{d \sigma}{d t d M_{X}^{2}}$ was measured in the region $0.024<-t<0.234(\mathrm{GeV} / c)^{2}$, $0<M^{2}<0.12 s$, and $(105<s<752) \mathrm{GeV}^{2}$, and a single peak in $M_{X}^{2}$ was identified.

Low-mass diffraction dissociation (DD) of protons, single

$$
p p \rightarrow p X
$$

and double, are among the priorities at the LHC.

For the compact muon solenoid (CMS) Collaboration, the SDD mass coverage is presently limited to some $10 \mathrm{GeV}$. With the Zero Degree Calorimeter (ZDS), this could be reduced to smaller masses, in case the SDD system produces very forward neutrals, e.g. a $N^{*}$ decaying into a fast leading neutron. Together with the $\mathrm{T} 2$ detectors of TOTEM, SDD masses down to $4 \mathrm{GeV}$ could be covered. This is not the case until TOTEM trigger (data acquisition) systems are combined together with the CMS ones. This is not likely before the year 2012 shutdown. In principle, ATLAS can do similar improvements, since the LHC layout at the distance of our proposed forward shower counters' (FSC) locations is similar. ALICE and LHCb have different beam arrangements, but their acceptances for central diffraction (double pomeron exchange) was also investigated, see, e.g., [7]).
While high-mass diffraction dissociation (DD) receives much attention, mainly due to its relatively easy theoretical treatment within the triple Reggeon formalism [8-11] and successful reproduction of the data $[8,12]$, this is not the case for low-masses, which are beyond the range of perturbative quantum chromodynamics (QCD). The forthcoming measurements at the LHC urge a relevant theoretical understanding and treatment of low-mass DD, which essentially has both spectroscopic and dynamic aspects. The low-mass $M_{X}$ spectrum is rich of nucleon resonances. Their discrimination is a difficult experimental task, and theoretical predictions of the appearance of the resonances depending on $s, t$, and $M$ is also very difficult since, as mentioned, perturbative QCD, or asymptotic Regge pole formulas, are of no use here. With this paper, we try to partially fill this gap, attacking the problem by means of a dual-Regge approach to the inelastic form factor (production amplitude) in which nonlinear Regge trajectories play an essential role.

We start with single diffraction dissociation (SDD). Generalization to double diffraction dissociation (DDD) is straightforward.

Diffraction, elastic and inelastic, in the LHC energy range is dominated by a single Pomeron exchange in the $t$ channel (see e.g., $[13,14]$ ), enabling the use of Regge factorization, Fig. 1. Accordingly, the knowledge of two vertices and the Regge propagator is essential for the construction of the scattering amplitude. Relying on the known properties of the elastic proton-Pomeron-proton vertex, and by adopting a simple supercritical Pomeron pole exchange (propagator) in the $t$ channel, we concentrate on the construction of a proper inelastic protonPomeron- $M_{X}$ vertex, the central object of our study. The solution of this problem, to a large extent, became possible due to the similarity between the inelastic $\gamma^{*} p \rightarrow M_{x}$ and Pomeron + proton $\rightarrow M_{x}$ vertices. We will extensively use the earlier results on the $\gamma^{*} p \rightarrow M_{x}$ transition, successfully applied to the JLab data $[15,16]$ in constructing the lower, 

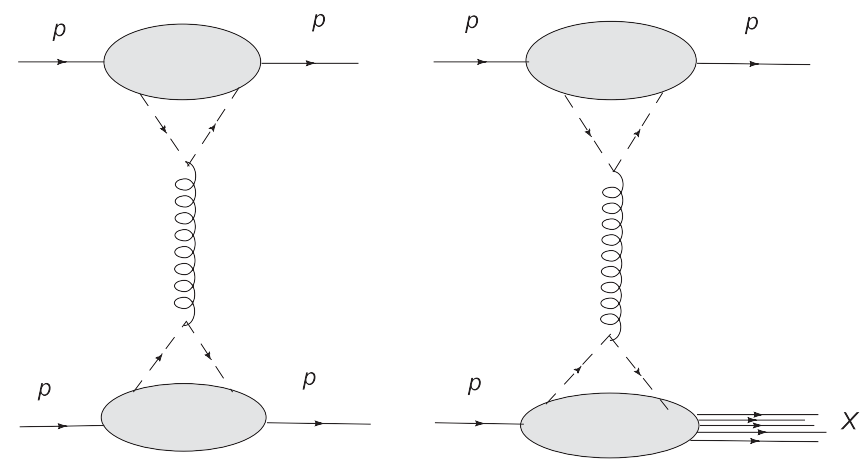

FIG. 1. Elastic scattering (left panel) and diffraction dissociation (right panel) in a model with a Pomeron exchange coupled to the proton by quarks.

Pomeron + proton $\rightarrow M_{x}$ vertices of Fig. 1, right panel. In doing so, we draw a parallel between the virtual photon and the Pomeron. They are similar, $W_{2}\left(q^{2}, s\right)_{\gamma^{*} p \rightarrow}$ $N_{i}^{*}, \Delta($ at JLab $) \rightarrow W_{2}\left(t, M^{2}\right)_{P p \rightarrow N^{*}}$ (at the LHC), apart from their opposite $C$ parities, and, of course, one should remember about the changes in kinematics: the photon virtuality (e.g., at the JLab) $Q^{2}$, etc. of Fig. 2 here becomes the squared momentum transfer $t$ of Fig. 1.

The unknown inelastic form factor of the type shown in Fig. 2, by the optical theorem, is related to the imaginary part of the forward $\gamma^{*}(P)-p$ scattering amplitude. Following Refs. [15-17], we use a dual amplitude for this reaction, in its low-energy (here: missing mass) resonance region, dominated by the contribution of relevant direct-channel trajectories. The correct choice of these trajectories is a crucial point in our approach. In the case of $\gamma^{*} p$ scattering (e.g., JLab), these were the $N^{*}$ and $\Delta$ trajectories, see Refs. [15-17]. Here, instead, by quantum numbers, the relevant direct-channel trajectory is that of the proton, to be parametrized in Sec. V.

In principle, one could proceed by counting the resonances one-by-one; however, apart from the technical complexity of counting single resonances, there is also a conceptual one: Regge trajectories and, more generally, dual models comprise the dynamics in a complete and continuous way, thus opening the way to study and relate different reactions in any kinematical region. Examples are finite mass sum rules, contained in the present formalism automatically. One more important point: the advantage of

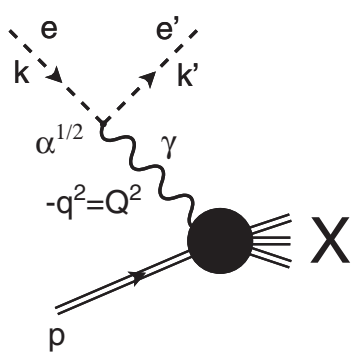

FIG. 2. Virtual photon + proton $\rightarrow M_{X}$ transition. using the dual-Regge model with nonlinear Regge trajectory presented in this paper over a one-to-one account for the particular resonances is that it automatically takes care of the relative weight of each resonance, and extrapolates to higher masses, with a limited number of resonances on any trajectory.

\section{ELASTIC SCATTERING}

The $p p$ scattering amplitude corresponding to Fig. 1 (left) is [10]

$$
A(s, t)=-\beta^{2}\left[f^{u}(t)+f^{d}(t)\right]^{2}\left(s / s_{0}\right)^{\alpha_{P}(t)-1} \frac{1+e^{-i \pi \alpha_{P}(t)}}{\sin \pi \alpha_{P}(t)},
$$

where $f^{u}(t)$ and $f^{d}(t)$ are the amplitudes for the emission of $u$ and $d$ valence quarks by the nucleon, $\beta$ is the quarkPomeron coupling, to be determined below; $\alpha_{P}(t)$ is a vacuum Regge trajectory. It is assumed [10] that the Pomeron couples to the proton via quarks like a scalar photon. Thus, the unpolarized elastic $p p$ differential cross section is

$$
\frac{d \sigma}{d t}=\frac{\left[3 \beta F^{p}(t)\right]^{4}}{4 \pi \sin ^{2}\left[\pi \alpha_{P}(t) / 2\right]}\left(s / s_{0}\right)^{2 \alpha_{P}(t)-2} .
$$

The norm $\beta$ appearing in Eq. (2) was found in Ref. [10] from the forward elastic scattering, $d \sigma / d t \approx 80 \mathrm{mb} / \mathrm{GeV}^{2}$ at $\sqrt{s}=23.6$ and $30.8 \mathrm{GeV}$, resulting, at unite Pomeron intercept, $\alpha_{P}(0)=1$, in $\beta^{4} /(4 \pi) \approx 1 \mathrm{mb} / \mathrm{GeV}^{2}$ [10].

To account for the rise of the cross sections, following the model and fits of Donnachie and Landshoff (see [13] and earlier references therein), we use a Pomeron trajectory whose intercept is slightly beyond one, namely, $\alpha_{P}(0)=1.08$ providing for excellent fits to the total cross sections [13]. However, the extrapolation with such an intercept and input value of $\beta$ strongly overshoots the elastic forward cross section measured at higher energies, e.g. $\sqrt{s}=1800 \mathrm{GeV}$ [18]. There are several reasons for this inconsistency. One is that, at the normalization point, 23.6 or $30.6 \mathrm{GeV}$, the contribution from secondary Reggeons, and/or a constant background should be included. In what follows, we use the Pomeron trajectory of the form (see [13]) $\alpha_{P}(t)=1.08+0.25 t$, and consequently relax the above norm of $\beta$. Instead, it will be included in the overall normalization factor of the amplitude/cross section $A_{0}$, that absorbs also the parameter $a$ of Eq. (17), from Section VI.

Another important issue is the neglect of absorption (unitary) corrections. We intend to come back in a forthcoming investigation to the study of the role of the subleading Reggeons and of the absorption corrections.

A dipole form can be used for the form factor

$$
F^{p}(t)=\frac{4 m^{2}-2.9 t}{4 m^{2}-t} \frac{1}{(1-t / 0.71)^{2}},
$$

where $m$ is the proton mass. 


\section{SINGLE DIFFRACTION DISSOCIATION (SDD)}

In single diffraction dissociation, Eq. (1), a system $X$ with a missing mass $M_{X}$ is produced at small $|t|$. At sufficiently large $s / M_{X}^{2}$, which is the case at the LHC, the process is dominated by a Pomeron exchange. This case was treated in Ref. [10] for missing masses beyond the resonance region, and in Ref. [19] in the resonance region. For large missing masses, the triple Regge limit applies [9,12,20,21]. Although large- $M_{X}$ diffraction dissociation is outside the scope of the present paper, we mention it below, in particular, in connection with duality relations called finite mass sum rules, that relate low- and high-missing mass dynamics.

Similar to the case of elastic scattering (Sec. II), the double differential cross section for the reaction (1), by Regge factorization, can be written as

$$
\begin{aligned}
\frac{d^{2} \sigma}{d t d M_{X}^{2}} \sim & \frac{9 \beta^{4}\left[F^{p}(t)\right]^{2}}{4 \pi \sin ^{2}\left[\pi \alpha_{P}(t) / 2\right]}\left(s / M_{X}^{2}\right)^{2 \alpha_{P}(t)-2} \\
& \times\left[\frac{W_{2}}{2 m}\left(1-M_{X}^{2} / s\right)-m W_{1}\left(t+2 m^{2}\right) / s^{2}\right],
\end{aligned}
$$

where $W_{i}, i=1,2$ are related to the structure functions of the nucleon and $W_{2} \gg W_{1}$. For high $M_{X}^{2}$, the $W_{1,2}$ are Regge-behaved, while for small $M_{X}^{2}$ their behavior is dominated by nucleon resonances. Thus, the behavior of (5) in the low missing mass region to a large extent depends on the transition form factors or resonance structure functions. The knowledge of the inelastic form factors (or transition amplitudes) is crucial for the calculation of low-mass diffraction dissociation from Eq. (5). We introduce these transition amplitudes in the next section.

At large $s$ (the LHC energies), one can safely neglect terms $M_{X}^{2} / s$ and $\left(t+2 m^{2}\right) / s$ in Eq. (5). Furthermore, we have replaced the familiar form of the signature factor in the amplitude, $\frac{1+e^{-i \pi \alpha_{P}(t)}}{\sin \pi \alpha_{P}(t)}$, used in [10], by a simple exponential one $e^{-i \pi \alpha_{P}(t) / 2}$. For the proton elastic form factor $F^{p}(t)$, Eq. (4), we use a dipole form

$$
F^{p}(t)=(1-t / 0.71)^{-2}
$$

(note that here we neglect the first factor of Eq. (4), producing a break in the small $|t|$ behavior of the elastic differential cross section).

Hence Eq. (5), in the LHC energy region simplifies to:

$$
\frac{d^{2} \sigma}{d t d M_{X}^{2}} \approx \frac{9 \beta^{4}\left[F^{p}(t)\right]^{2}}{4 \pi}\left(s / M_{X}^{2}\right)^{2 \alpha_{P}(t)-2} \frac{W_{2}}{2 m} .
$$

Equations (5) and (7) do not contain the elastic scattering limit because the inelastic form factor $W_{2}\left(M_{X}, t\right)$ has no elastic form factor limit $F(t)$ as $M_{X} \rightarrow m$. This problem is similar to the $x \rightarrow 1$ limit of the deep inelastic structure function $F_{2}\left(x, Q^{2}\right)$. The elastic contribution to SDD should be added separately, as discussed below in Sec. VI. To be sure, we eliminate in the present work this region by imposing $M_{X}^{2}>2 \mathrm{GeV}^{2}$.

\section{TRANSITION FORM FACTORS}

The one-by-one account for single resonances is a possible, although not efficient for the calculation of the SDD cross section, to which, at low missing masses, a sequence of many resonances contribute. The definition and identification of these resonances is not unique; moreover with increasing masses (still within "low-mass diffraction"), they gradually disappear. Similar to the case of electroproduction, the (dis)appearance of resonances in the cross section depends on two variables, their mass and the virtuality or the "probe" (photon with $Q^{2}$ in electroproduction and Pomeron with $t$ in SDD). The finite widths of the resonances can be introduced by a replacement [22]:

$$
\delta\left(W^{2}-m_{N}^{2}\right) \approx \frac{1}{2 m_{R} \pi} \frac{\Gamma_{R} / 2}{\left(W-m_{R}\right)^{2}+\Gamma_{R}^{2} / 4},
$$

where $\Gamma_{R}$ is the widths of the resonance. At a resonance, $W=m_{R}$, the peak goes as high as $1 /\left(\pi m_{R} \Gamma_{R}\right)$.

A way to account for many resonances was suggested in paper [15], based on the ideas of duality with a limited number of resonances lying on nonlinear Regge trajectories. This approach was used [16] in a kinematically complete analysis of the CLAS data from the JLab on the proton structure function. The similarity between electroproduction of resonances (e.g., at JLab) and low-mass SDD is the key point of our model. The inelastic form factor (transition amplitude), the main ingredient of the model, is constructed by analogy with the nucleon resonances electroproduction amplitude. In both cases, many resonances overlap and their appearance depends both on the reaction energy, which is replaced here by missing mass $\left(s \Rightarrow M_{X}^{2}\right)$, and virtuality of the incident probe, which is replaced here by the Pomoron's momentum transfer $\left(Q^{2} \Rightarrow-t\right)$. This interplay makes the problem complicated and interesting.

\section{A. Dual amplitude with Mandelstam analyticity}

The main idea behind the present work is the Regge-dual connection between the inelastic form factor, Fig. 2, appearing in the lower vertex of Fig. 1, and the directchannel, low energy (here: missing mass) dual amplitude, as illustrated in Fig. 3.

Figure 3 shows the connection between the inelastic form factor (structure function) appearing in the lower vertex of the left panel of Fig. 1, via duality, unitarity (generalized optical theorem) and Veneziano-duality, and its direct-channel, resonance decomposition (rightmost term of Fig. 3).

The invariant on-shell scattering dual amplitude with Mandelstam analyticity (DAMA), applicable both to the diffractive and nondiffractive components, reads [15-17,23]

$$
D(s, t)=\int_{0}^{1} d z\left(\frac{z}{g}\right)^{-\alpha_{s}\left(s^{\prime}\right)-1}\left(\frac{1-z}{g}\right)^{-\alpha_{t}\left(t^{\prime}\right)-1},
$$




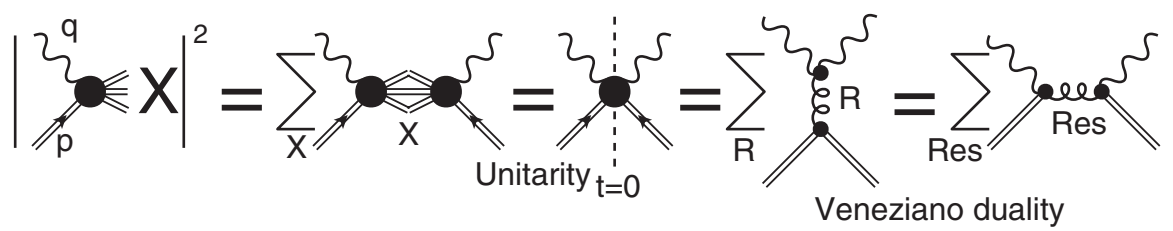

FIG. 3. Connection, through unitarity (generalized optical theorem) and Veneziano-duality, between the inelastic form factor and the sum of direct-channel resonances.

where $s^{\prime}=s(1-z), t^{\prime}=t z, g$ is a parameter, $g>1$, and $s$, $t$ are the Mandelstam variables.

For $s \rightarrow \infty$ and fixed $t$ it has the following Regge asymptotic behavior:

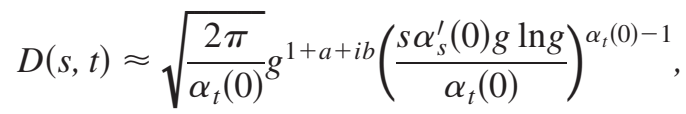

where $a=\operatorname{Re} \alpha\left(\frac{\alpha_{t}(0)}{\alpha_{s}^{\prime}(0) \ln g}\right)$ and $b=\operatorname{Im} \alpha\left(\frac{\alpha_{t}(0)}{\alpha_{s}^{\prime}(0) \ln g}\right)$.

Contrary to the Veneziano model, DAMA [23] not only allows for, but rather requires the use of, nonlinear complex trajectories providing the resonance widths via the imaginary part of the trajectory, and, in a special case of restricted real part of the trajectory, resulting in a finite number of resonances. More specifically, the asymptotic rise of the trajectories in DAMA is limited by the important upper bound

$$
\left|\frac{\alpha_{s}(s)}{\sqrt{s} \ln s}\right| \leq \text { const, } \quad s \rightarrow \infty .
$$

The pole structure of DAMA is similar to that of the Veneziano model, except that multiple poles appear on daughter levels [15-17,23],

$$
D(s, t)=\sum_{n=0}^{\infty} g^{n+1} \sum_{l=0}^{n} \frac{\left[-s \alpha_{s}^{\prime}(s)\right]^{l} C_{n-l}(t)}{\left[n-\alpha_{s}(s)\right]^{l+1}},
$$

where $C_{n}(t)$ is the residue, whose form is fixed by the $t$-channel Regge trajectory (see [23])

$$
C_{l}(t)=\frac{1}{l !} \frac{d^{l}}{d z^{l}}\left[\left(\frac{1-z}{g}\right)^{-\alpha_{t}(t z)}\right]_{z=0} .
$$

The presence of the multipoles, Eq. (10), does not contradict the theoretical postulates. On the other hand, they can be removed without any harm to the dual model by means the so-called Van der Corput neutralizer [23], resulting in a "Veneziano-like" pole structure:

$$
D(s, t)=\sum_{n=0}^{\infty} \frac{C_{n}}{n-\alpha_{s}(s)} .
$$

We disregard the symmetry (spin and isospin) properties of the problem, concentrating on its dynamics.

The main problem is how to introduce $Q^{2}$-dependence in the dual model, matching its Regge asymptotic behavior and pole structure to standard forms known from the literature. (This is the famous problem of the off-mass-shell continuation of the $S$ matrix.) Note that any correct identification of this $Q^{2}$-dependence in a single asymptotic limit of the dual amplitude, by duality, will extend it to other kinematical regions. In Refs. [15-17], a solution combining Regge behavior and Bjorken scaling limits of the structure functions (or $Q^{2}$-dependent $\gamma^{*} p$ cross sections) was suggested (for an alternative solution, see Ref. [24]).

Let us remind that below $Q^{2}$ (photon virtuality in electroproduction) will be replaced by $-t$ (Pomeron "virtuality"), and $s$ will be replaced by $M_{X}^{2}$ (the direct, $P p$ channel "energy").

\section{B. Dual-Regge model of the inelastic form factors (transition amplitudes)}

For our purposes, i.e. for low-mass SDD, the directchannel pole decomposition of the dual amplitude (12) is relevant. Anticipating its application in SDD, we write it as [25]

$$
A\left(M_{X}^{2}, t\right)=a \sum_{n=0,1, \ldots} \frac{f(t)^{2(n+1)}}{2 n+0.5-\alpha\left(M_{X}^{2}\right)},
$$

where $\alpha\left(M_{X}^{2}\right)$ is a nonlinear Regge trajectory in the Pomeron-proton system, $t$ is the squared transfer momentum in the $P p \rightarrow P p$ reaction, and $a$ is the normalization factor, which will be absorbed together with $\beta$ in the overall normalization coefficient $A_{0}$ to be fitted to the data, see Sec. VI.

The form factor $f(t)$ appearing in the $P p \rightarrow P p$ system should not be confused with $F^{p}(t)$ in Eq. (1) (the $p p P$ vertex). It is fixed by the dual model [15-17,24], in particular, by the compatibility of its Regge asymptotics with Bjorken scaling [15-17] and reads

$$
f(t)=\left(1-t / t_{0}\right)^{-2},
$$

where $t_{0}$ is a parameter to be fitted to the data, for example, by comparing the hight of the resonance peaks for different $t$. However, since for the moment we have no data on differential SDD cross section, for simplicity we set $t_{0}=0.71 \mathrm{GeV}^{2}$, as in the proton elastic form factor, Eq. (4).

Notice that in Eq. (13), this form factor enters with a power $2(n+1)$ strongly damping higher spin resonances contributions [26]. 
The inelastic form factor in diffraction dissociation is similar to that in $\gamma^{*} p$, treated in Ref. [16], up to the replacement of the photon by a Pomeron, whose parity is different from that of the photon. As a consequence, we have a single direct-channel resonance trajectory, that of the proton, plus the exotic, nonresonance trajectory providing the background, dual to the Pomeron exchange in the cross channel. The proton trajectory was studied in detail in Ref. [27] and will be introduced in the next section.

Then we proceed, (see for example [24]):

$$
\begin{aligned}
\nu W_{2}\left(M_{X}^{2}, t\right) & =F_{2}(x, t) \\
& =\frac{4(-t)(1-x)^{2}}{\alpha\left(M_{x}^{2}-m^{2}\right)\left(1+4 m^{2} x^{2} /(-t)\right)^{3 / 2}} \operatorname{Im} A\left(M_{X}^{2}, t\right),
\end{aligned}
$$

where $\alpha$ is a fine structure constant, $\nu$ is defined via $2 m \nu=M_{x}^{2}-m^{2}-t$, and $x=\frac{-t}{2 m \nu}$ is the Bjorken variable. Thus, finally we have

$$
\begin{aligned}
& \frac{W_{2}\left(M_{X}^{2}, t\right)}{2 m} \\
& \quad=\frac{4 x(1-x)^{2}}{\alpha\left(M_{x}^{2}-m^{2}\right)\left(1+4 m^{2} x^{2} /(-t)\right)^{3 / 2}} \operatorname{Im} A\left(M_{X}^{2}, t\right) .
\end{aligned}
$$

The imaginary part of the transition amplitude reads

$$
\begin{aligned}
& \operatorname{Im} A\left(M_{X}^{2}, t\right) \\
& \quad=a \sum_{n=0,1, \ldots} \frac{[f(t)]^{2(n+1)} \operatorname{Im} \alpha\left(M_{x}^{2}\right)}{\left(2 n+0.5-\operatorname{Re} \alpha\left(M_{X}^{2}\right)\right)^{2}+\left(\operatorname{Im} \alpha\left(M_{X}^{2}\right)\right)^{2}} .
\end{aligned}
$$

Next, we insert the proton trajectory $\alpha\left(M_{X}^{2}\right)$ into Eq. (17), and subsequently into Eq. (7). The explicit expression for the proton trajectory and the values of the parameters are presented in the next section. For more details, see also Ref. [27].

\section{THE PROTON TRAJECTORY IN THE $M_{X}^{2}$-CHANNEL}

The Pomeron-proton channel, $P p \rightarrow M_{X}^{2}$ (see the lower part of Fig. 1, right panel) couples to the proton trajectory, with the $I\left(J^{P}\right)$ resonances: $1 / 2\left(5 / 2^{+}\right), F_{15}$, $m=1680 \mathrm{MeV}, \Gamma=130 \mathrm{MeV} ; 1 / 2\left(9 / 2^{+}\right), H_{19}, m=$ $2200 \mathrm{MeV}, \quad \Gamma=400 \mathrm{MeV}$; and $1 / 2\left(13 / 2^{+}\right), \quad K_{1.13}$, $m=2700 \mathrm{MeV}, \Gamma=350 \mathrm{MeV}$. The status of the first two is firmly established [28], while the third one, $N^{*}(2700)$, is less certain, with its width varying between $350 \pm 50$ and $900 \pm 150 \mathrm{MeV}$ [28]. Still, with the stable proton included, we have a fairly rich trajectory, $\alpha\left(M^{2}\right)$, whose real part is shown in Fig. 4.

Despite the seemingly linear form of the trajectory, it is not that: the trajectory must contain an imaginary part corresponding to the finite widths of the resonances on it.
The nontrivial problem of combining the nearly linear and real function with its imaginary part was solved in Ref. [27] by means of dispersion relations.

We use the explicit form of the trajectory derived in Ref. [27], ensuring correct behavior of both its real and imaginary parts. The imaginary part of the trajectory can be written in the following way:

$$
\operatorname{Im} \alpha(s)=s^{\delta} \sum_{n} c_{n}\left(\frac{s-s_{n}}{s}\right)^{\lambda_{n}} \cdot \theta\left(s-s_{n}\right),
$$

where $\lambda_{n}=\mathcal{R} e \alpha\left(s_{n}\right)$. Equation (18) has the correct threshold behavior, while analyticity requires that $\delta<1$. The boundedness of $\alpha(s)$ for $s \rightarrow \infty$ follows from the condition that the amplitude, in the Regge form, should have no essential singularity at infinity in the cut plane.

The real part of the proton trajectory is given by

$$
\mathcal{R} e \alpha(s)=\alpha(0)+\frac{s}{\pi} \sum_{n} c_{n} \mathcal{A}_{n}(s),
$$

where

$$
\begin{aligned}
\mathcal{A}_{n}(s) \\
=\frac{\Gamma(1-\delta) \Gamma\left(\lambda_{n}+1\right)}{\Gamma\left(\lambda_{n}-\delta+2\right) s_{n}^{1-\delta} 2} F_{1}\left(1,1-\delta ; \lambda_{n}-\delta+2 ; \frac{s}{s_{n}}\right) \theta\left(s_{n}-s\right) \\
\quad+\left\{\pi s^{\delta-1}\left(\frac{s-s_{n}}{s}\right)^{\lambda_{n}} \cot [\pi(1-\delta)]\right. \\
\left.\quad-\frac{\Gamma(-\delta) \Gamma\left(\lambda_{n}+1\right) s_{n}^{\delta}}{s \Gamma\left(\lambda_{n}-\delta+1\right)}{ }_{2} F_{1}\left(\delta-\lambda_{n}, 1 ; \delta+1 ; \frac{s_{n}}{s}\right)\right\} \theta\left(s-s_{n}\right) .
\end{aligned}
$$

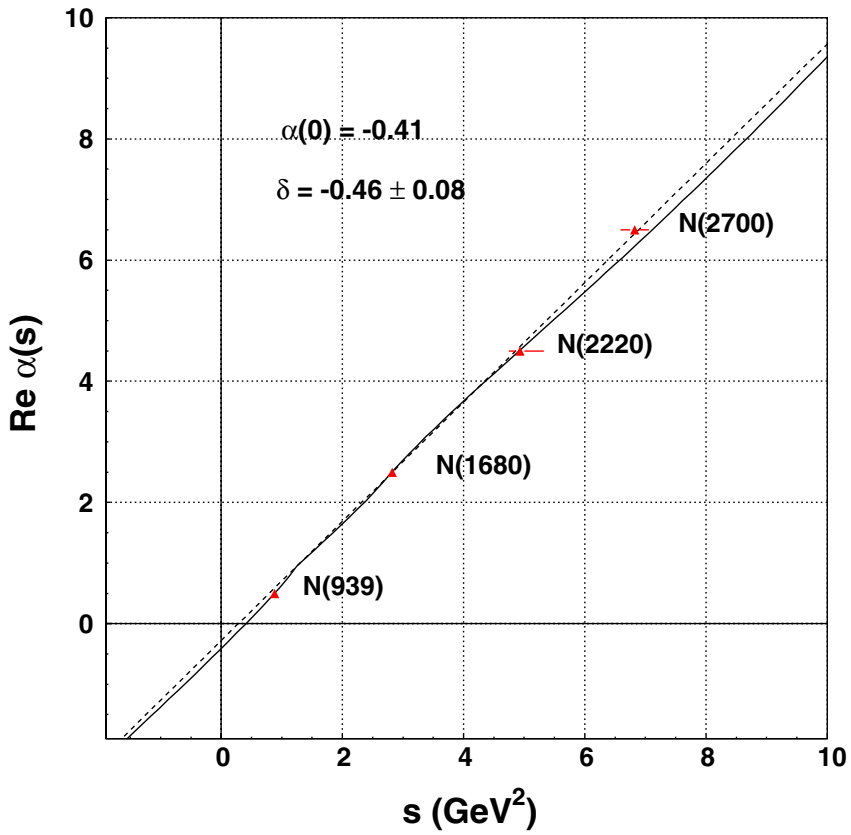

FIG. 4 (color online). The real part of the proton Regge trajectory. The dashed line corresponds to the result of a linear fit, the solid line is the fit from [27]. 
As already mentioned, the proton trajectory, also called $N^{+}$trajectory [27], contains the baryons $N(939) \frac{1}{2}^{+}$, $N(1680) \frac{5}{2}^{+}, N(2220) \frac{9}{2}^{+}$and $N(2700) \frac{13}{2}^{+}$[28]. In the fit, the input data are the masses and widths of the resonances. The quantities to be determined are the parameters $c_{n}, \delta$ and the thresholds $s_{n}$. Following [27], we set $n=1,2, x$ and $s_{1}=\left(m_{\pi}+m_{N}\right)^{2}=1.16 \mathrm{GeV}^{2}, s_{2}=2.44 \mathrm{GeV}^{2}$ and $s_{x}=11.7 \mathrm{GeV}^{2}$.

Other parameters of the trajectory, obtained in the fit, are summarized below: $\alpha(0)=-0.41, \delta=-0.46 \pm$ $0.07, \quad c_{1}=0.51 \pm 0.08, \quad c_{2}=4.0 \pm 0.8$ and $c_{x}=$ $(4.6 \pm 1.7) \times 10^{3}$. Taking the central values of these parameters, we obtain the following values for the $\lambda$ 's: $\lambda_{1}=0.846, \lambda_{2}=2.082, \lambda_{x}=11.177$.

The fit is fairly good: $\chi^{2} /$ d.o.f $=1.15$, see Figs. 4 and 5 . In the mass range, the parameters of the trajectory were fitted to the data, i.e. $M_{X}^{2} \leq 8 \mathrm{GeV}^{2}$; this is the most realistic proton trajectory we know from the literature. Nevertheless, care should be taken if used outside this range. As long as we are within our applicability range, the sum over resonances in Eq. (13) is restricted to 4 resonances $(n=0,3)$, but in the imaginary part of the transition amplitude, Eq. (17) we consider the contributions only from three of these resonances, since for the lowest resonance, i.e. for the proton, $n=0$, the imaginary part vanishes, $\operatorname{Im} \alpha=0$, producing an infinitely narrow and high peak.

The elastic contribution, $p P \rightarrow p P$ will be discussed in the next section, see also [29,30]. However, it can be assumed that outside the elastic peak, $2 \mathrm{GeV}^{2} \leq M_{X}^{2} \leq 8 \mathrm{GeV}^{2}$,

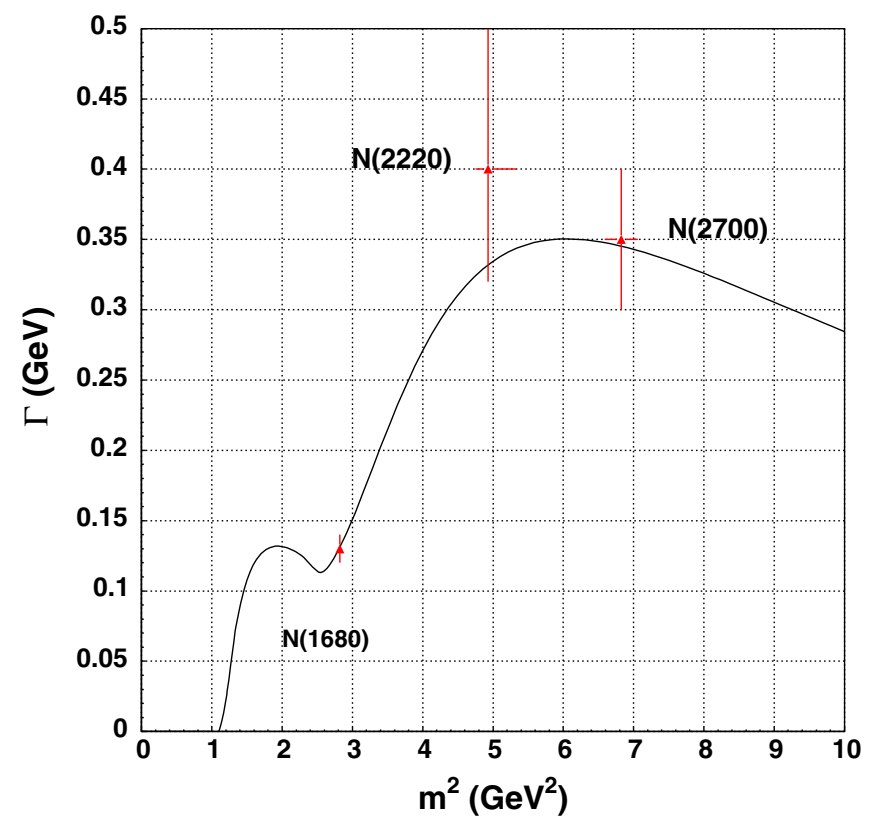

FIG. 5 (color online). The widths of the resonances, $\Gamma=$ $\frac{\operatorname{Im} \alpha\left(M^{2}\right)}{M \mathcal{R} e \alpha^{\prime}\left(M^{2}\right)}$, appearing on the proton trajectory, calculated and fitted in [27]. this distribution can be neglected, because the dominant part comes from the nearest resonance.

Thus, we obtain:

$$
\begin{aligned}
\operatorname{Im} A\left(M_{X}^{2}, t\right)= & a \sum_{n=1,3}[f(t)]^{2(n+1)} \\
& \times \frac{\operatorname{Im} \alpha\left(M_{X}^{2}\right)}{\left(2 n+0.5-\operatorname{Re} \alpha\left(M_{X}^{2}\right)\right)^{2}+\left(\operatorname{Im} \alpha\left(M_{X}^{2}\right)\right)^{2}} .
\end{aligned}
$$

Note that the contribution from each subsequent resonance of the proton trajectory is suppressed by a factor $f(t)^{2}$ compared with the previous one.

Apart from the well-established proton trajectory, with a sequence of four particles on it, there is a prominent resonance $I=1 / 2, J=1 / 2^{+}$with mass $1440 \mathrm{MeV}$, known as the Roper resonance. It is wide, the width being nearly one quarter of its mass. The Roper resonance may appear on the daughter trajectory of $N^{*}$ treated above, although its status is still disputable. In the Appendix, we consider the possible contribution from the single Roper resonance by means of a separate Breit-Wigner term.

\section{RESULTS}

Figure 6 shows the behavior of the imaginary part of the transition amplitude, Eq. (20), proportional to the transition form factor as in Fig. 2, or lower vertex in Fig. 1 (right panel). It shows the resonance structure corresponding to the proton trajectory, to be translated into the cross sections via Eq. (7) and (16), with the results shown below, Eq. (21).

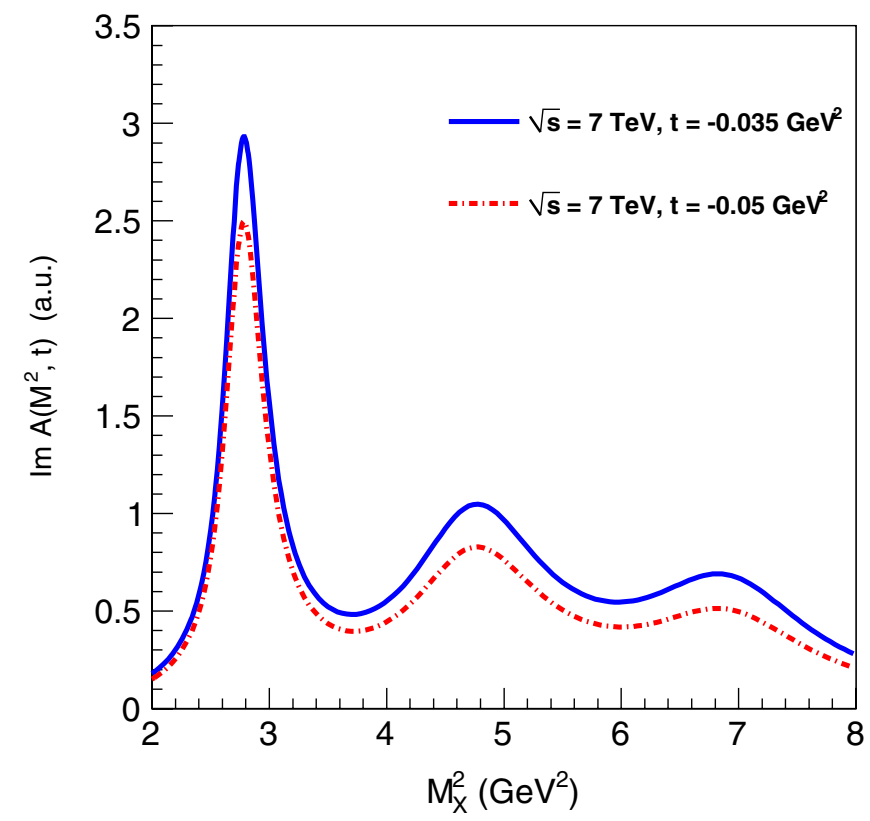

FIG. 6 (color online). Imaginary part of the amplitude $A\left(M_{X}^{2}, t\right)$, Eq. (20). 
One can see that the imaginary part of transition amplitude decreases with growing $|t|$, due to the dipole form factor $f(t)^{2(n+1)}$. Furthermore for each fixed $t$ the relative contribution of higher resonances decreases, because of the suppression factor $f(t)^{2}$ for every subsequent resonance. Such a behavior results from dual models.

Our final expression for the double differential cross section reads

$$
\frac{d^{2} \sigma}{d t d M_{X}^{2}}=A_{0}\left(\frac{s}{M_{X}^{2}}\right)^{2 \alpha_{P}(t)-2} \frac{x(1-x)^{2}\left[F^{p}(t)\right]^{2}}{\left(M_{x}^{2}-m^{2}\right)\left(1+\frac{4 m^{2} x^{2}}{-t}\right)^{3 / 2}} \sum_{n=1,3} \frac{[f(t)]^{2(n+1)} \operatorname{Im} \alpha\left(M_{X}^{2}\right)}{\left(2 n+0.5-\operatorname{Re} \alpha\left(M_{X}^{2}\right)\right)^{2}+\left(\operatorname{Im} \alpha\left(M_{X}^{2}\right)\right)^{2}} .
$$

Its over-all normalization depends on two factors, namely $\beta$, Eq. (7), and $a$, Eq. (13), but since they do not appear separately, we have combined them in a single factor, $A_{0}=\frac{9 a \beta^{4}}{\pi \alpha}$, to be fitted to the data.

Data on the integrated SDD cross sections for different $s$ are available from [4-6] (see also [31]). Unfortunately, these data points are not sufficient to fix this norm unambiguously and to discriminate uniquely the resonance contribution from the background. Furthermore, in the present model, applicable at the LHC, only the Pomeron trajectory is considered in the $t$ channel. At much lower energies, where data are available: Fermilab, ISR,...-secondary, nonleading trajectories give some contribution as well. They should be included in a future, more refined, analysis (fit) of these data.

To calculate the integrated SDD, we first take into account the contribution from the resonance region. This is done by integrating Eq. (21) in squared momentum transfer $t$ from $-\infty$ to 0 , and in the missing mass $M_{x}$ over the resonance region, $2 \mathrm{GeV}^{2}<M_{x}^{2}<8 \mathrm{GeV}^{2}$, where the contributions from the resonances, Eq. (7) dominate. We thus eliminate contributions from the region of the elastic peak, $M_{X}^{2}<2 \mathrm{GeV}^{2}$, that requires separate treatment (see [32]), and the high missing mass Reggebehaved region. By duality, to avoid "double counting,"

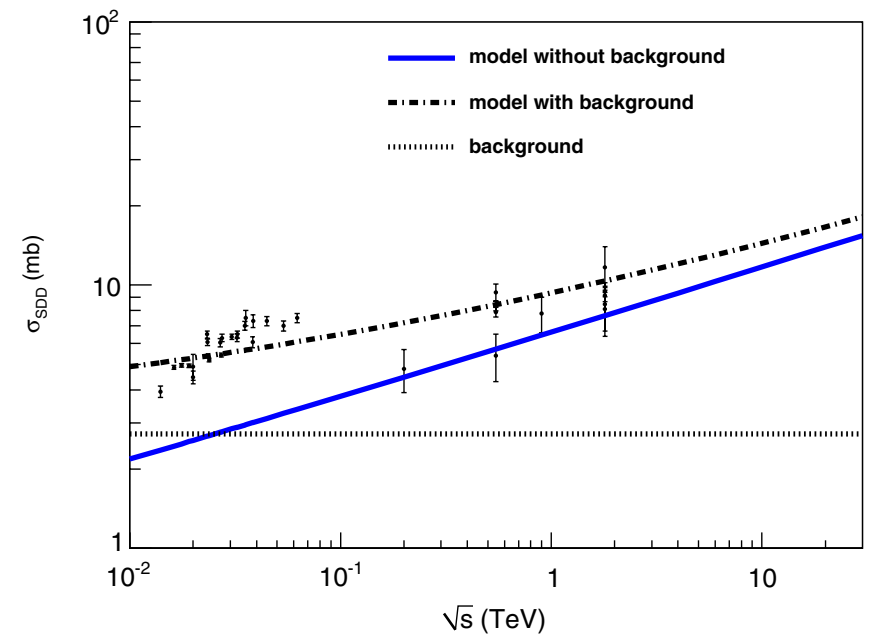

FIG. 7 (color online). Predicted integrated SDD cross section as a function of $s$ compared with the experimental data [4-6,35-40]; see also [31]. the latter should be accounted for automatically, provided the resonance contribution is included properly.

The results for the integrated SDD cross section are shown in Fig. 7. Without any background contribution, fits to the data give $A_{0}=977 \pm 5 \mathrm{mb} / \mathrm{GeV}^{2}$ with $\chi^{2} /$ d.o.f. $=25.7$. Better agreement with the data can be obtain by including a constant background, i.e. by adding a fitting parameter $b$ to the integrated SDD cross section. In this case, the fit gives $\chi^{2} /$ d.o.f. $=11.5$, with $A_{0}=506 \pm$ $23 \mathrm{mb} / \mathrm{GeV}^{2}$ and $b=2.72 \pm 0.13 \mathrm{mb}$. Lacking any reliable model for the background, we avoid complicated background parameterization. Our constant background gives about $20 \%$ contribution at LHC energy; that seems to be a reasonable number.

Having fixed the parameters of the model, we can now scrutinize the SDD cross section in more details. First, we calculate double differential cross section, Eq. (21), as a function of the missing mass for several fixed values of the momentum transfer $t$ and two representative LHC energies, 7 and $14 \mathrm{TeV}$. The results of such calculations are shown in Fig. 8.

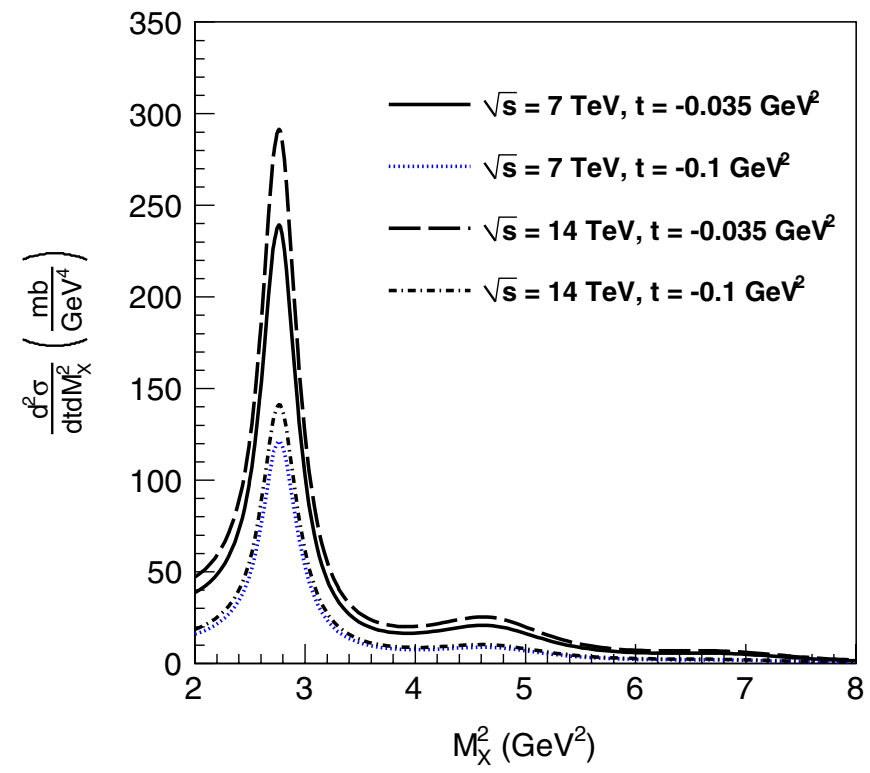

FIG. 8 (color online). Double differential cross section of SDD as a function of $M_{X}^{2}$ for several fixed values of $t$ and two representative LHC energies. 


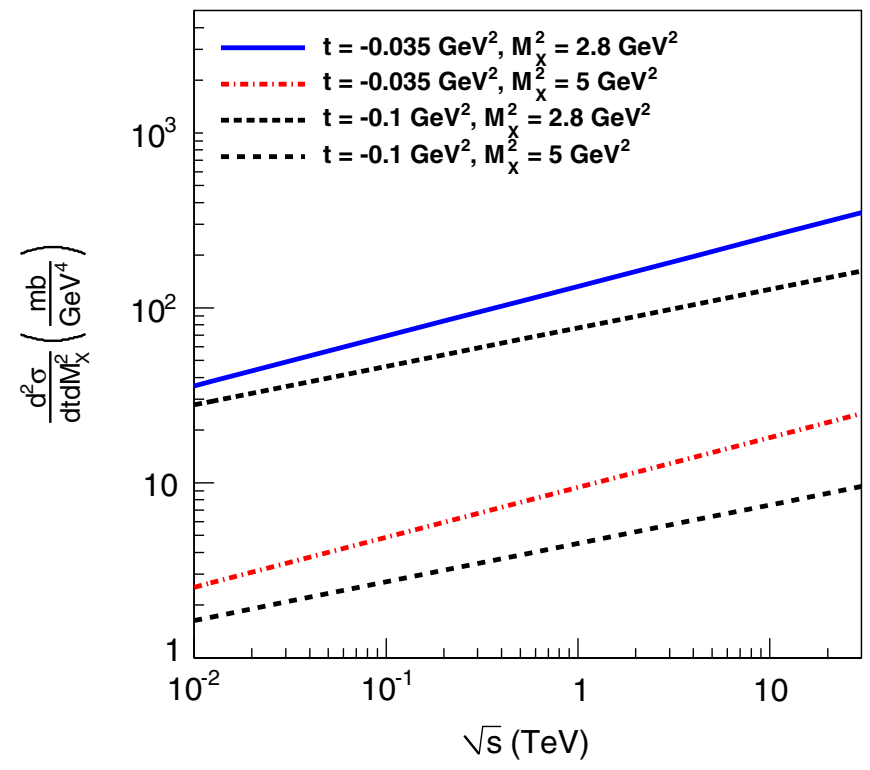

FIG. 9 (color online). Double differential cross section of SDD as a function of $s$ at fixed values of $t$ and $M_{X}^{2}$.

In Fig. 9, we show the energy dependence of the double differential cross section for several fixed values of $t$ and $M_{X}$. The rise of $\sigma_{\mathrm{SDD}}(s)$ is mainly determined by the supercritical Pomeron intercept $\alpha_{P}(0)$, and only weakly

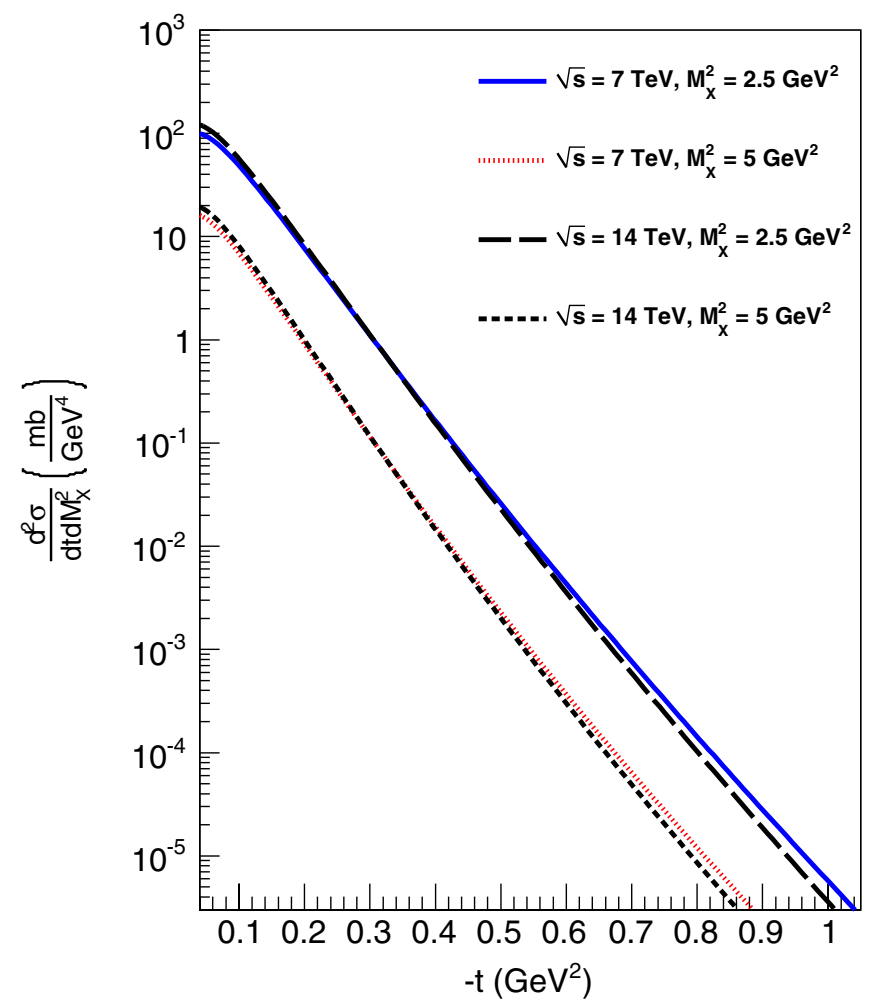

FIG. 10 (color online). Double differential cross section of SDD as a function of $t$ at fixed values of $s$ and $M_{X}^{2}$.

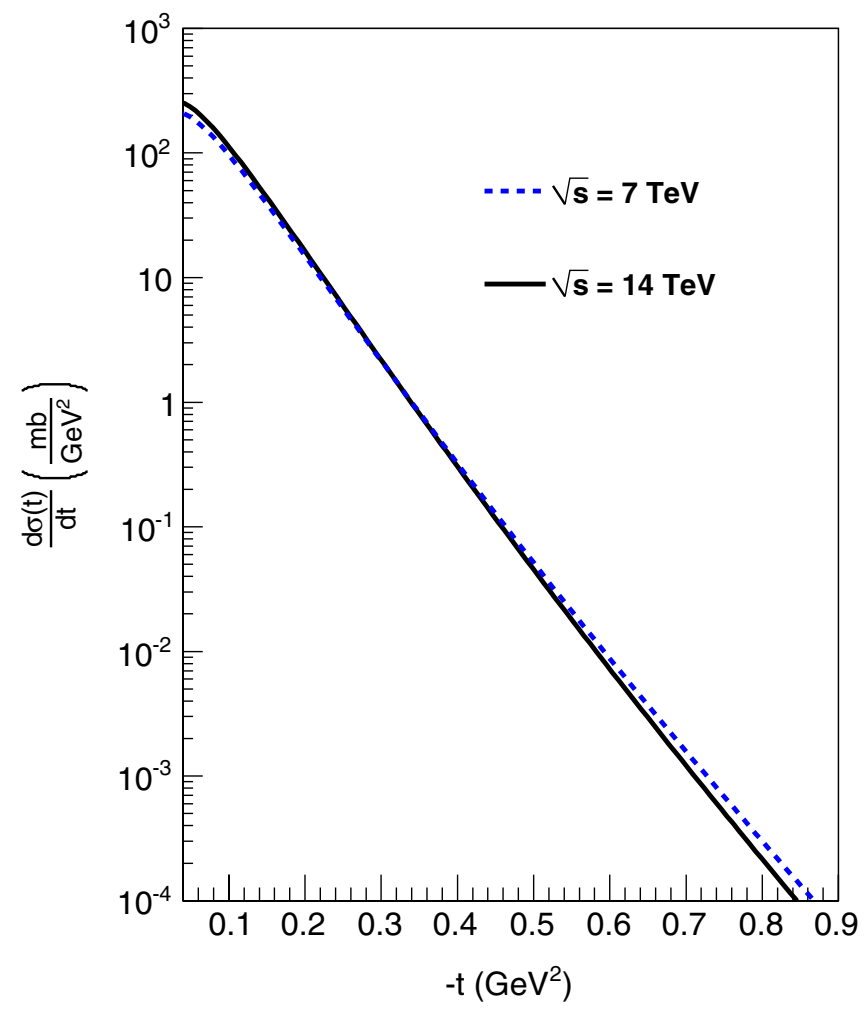

FIG. 11 (color online). Integrated cross section $d \sigma / d t$ as a function of $t$.

affected also by the details of the $t$ - dependence of the Pomeron trajectory.

The double differential cross sections as a function of $t$ for two representative LHC energies and several fixed values of $M_{X}$ are presented in Fig. 10. Figure 11 shows the $t$ - dependence of the differential cross section integrated in $M_{X}^{2}$ for representative LHC energies.

\section{CONCLUSIONS}

Let us briefly summarize the status of the present model and its credibility, including the way its parameters were fixed. As already mentioned, the normalization constant, $\beta$, discussed in Secs. II and III is absorbed by the overall norm $A_{0}$, together with the other normalization parameter $a$. The parameters of the Pomeron trajectory were determined [13] from $p p$ elastic and total cross section data. The form and the values of the parameters of the proton trajectory, that plays a crucial role in predicting the $M_{X}$ dependence, are fixed by spectroscopic data, see Sec. V. Finally, the overall norm $A_{0}$ is fixed from the comparison of calculated SDD cross section with the experimental data, with the following caveat: in the present model, applicable at the LHC, only the Pomeron trajectory contributes in the $t$ channel. At much lower energies (Fermilab, ISR,...), where data are available, apart from 
the Pomeron, secondary, nonleading trajectories contribute as well. We plan to include these in a future, more refined, study.

There is some freedom in the form and weight of the background. Its relative contribution can only be normalized to earlier measurements at the ISR or the Fermilab. For a better control, we compare our predictions with the experimental data [4-6] and theoretical estimates $[12,20,31]$. In any case, it follows from our model and the fits to the data that the background is fairly large: about $20 \%$ at the LHC. A dedicated study of various options for the background in SDD can be found in Ref. [32].

The elastic contribution, $p p \rightarrow p p$ is usually calculated and measured separately. There is no consistent theoretical prescription of any smooth transition from inelastic to elastic scattering, corresponding to the $x \rightarrow 1$ limit for the structure functions (see Ref. [32]).

There is an important point omitted in this short paper, namely, unitarity. As is well known (see e.g., [21]) any simple Regge pole model violates unitarity in the sense that the DD cross section asymptotically grows faster that the total cross section (it is obvious that no partial cross section can overshoot the total cross section). This longstanding problem was cured in various ways, the final answer being still open. In Refs. [8,12,20] unitarity is restored by a renormalization procedure. Without entering into details, here we only mention that a possible solution of this problem can be found by using a more realistic (and complicated) Pomeron singularity, for example, in the form of a double pole [33].

The model presented in this paper and the calculated cross sections, corrected for the efficiencies of relevant detectors will be used [34] in future measurements at the LHC.

As already mentioned in the Introduction, the prospects of measuring SDD at the LHC are promising, although some details still remain to be settled. For the CMS Collaboration, the SDD mass coverage is presently limited to some $10 \mathrm{GeV}$. Together with the T2 detectors of TOTEM, SDD masses down to $4 \mathrm{GeV}$ could be covered, provided the TOTEM trigger (data acquisition) system will be combined with the CMS ones. ALICE and LHCb have different beam arrangements, but their acceptances for central diffraction (double Pomeron exchange) was also investigated (see e.g., [7]). Measurements of the SDD events at the LHC are based on: (1) identifying a gap in forward rapidities in conjunction with a veto for any activity on the opposite side of the interaction point, or (2) detecting a diffractively scattered proton in a leading proton detector, such as the Roman Pots, and a coincident diffractively excited bunch of particles on the opposite side. The problem with both measurement strategies stems from the incomplete rapidity coverage of the base line detector systems at the LHC: the low mass,
$M<4 \mathrm{GeV}$, diffractively excited states are not seen. Without extra rapidity coverage below $M=4 \mathrm{GeV}$, both approaches to SDD identification fail. In case of purely rapidity gap-based method, the recorded cross section misses the SDD events with diffractive masses below $4 \mathrm{GeV}$. In case a leading proton is detected on one side of the intersection point (IP), one could, in principle, be sensitive to diffractive masses that correspond to the uncertainty in LHC beam energy. In practice, it is impossible to trigger for these events, and the low mass SDD events will be missed by this method as well. Detecting SDD events with high acceptance is essential for determining the total pp cross section in the so-called luminosity independent method based on using the Optical Theorem. The method bases on measuring the slope of the elastic cross section, extrapolating the slope to the optical point. Together with the overall inelastic rate (plus the ratio between the inelastic and elastic forward scattering amplitudes), the total pp cross section is obtained. The main uncertainty in this evaluation is due to the error in estimating the inelastic pp event rate. As shown by the authors of Ref. [7], the acceptance of basically all the LHC experiments can be substantially improved by adding forward detector systems (forward shower counters, FSCs) that register secondary interactions within the beam pipe due to particles-both electrically neutral and charged-emitted at very small scattering angles with respect to the beam direction. With the addition of FSCs, rapidity coverage of an LHC experiment can be extended down to SDD masses of the order of $1.2 \mathrm{GeV}$, i.e. down to the dominant $N^{*}$ states. FSCs are being currently installed in ALICE and CMS detectors, and they will provide the necessary added coverage of small mass forward systems at the LHC.

\section{ACKNOWLEDGMENTS}

We thank K. Goulianos and F. Paccanoni for useful discussions. R. O. would like to acknowledge his thanks to the Academy of Finland for support. O. K. is grateful to Rainer Schicker and A. G. Zagorodny for their support, and acknowledges the hospitality of the Bogolyubov Institute for Theoretical Physics (Kiev, Ukraine); his work was supported by the EU program "Difractive and Electromagnetic Processes at the LHC." The work of V. K. M. is partly supported by Contract No. FIS200801661 from MICINN (Spain), by the Generalitat de Catalunya Contract No. 2009SGR-1289, and by the European Community-Research Infrastructure Integrating Activity "Study of Strongly Interacting Matter" (HadronPhysics2, Grant Agreement No. 227431) under the Seventh Framework Programme of the EU. L. J. was supported by the National Academy of Sciences of Ukraine, Department of Astronomy and Physics, under the Grant "Matter at Extreme Condition". 


\section{APPENDIX: ROPER}

Apart from the well established protonic trajectory with a sequence of four particles, on it Sec. V, there is a prominent single resonance $I=1 / 2, J=1 / 2^{+}$with mass $1440 \mathrm{MeV}$, known as the Roper resonance [28]. It is wide, the width being nearly one fourth of its mass, its spectroscopic status being disputable. There is no room for the Roper resonance on the proton trajectory of Sec. V, although it could still be a member of proton's daughter trajectory. Waiting for a future better understanding of Roper's status, here we present the contribution to SDD cross section of a single Roper resonance, calculated from a simple Breit-Wigner formula:

$$
\begin{aligned}
& \operatorname{Im} A_{\text {incl.Roper }}\left(M_{X}^{2}, t\right) \\
& =a\left(\sum_{n=1,3} \frac{[f(t)]^{2(n+1)} \operatorname{Im} \alpha\left(M_{X}^{2}\right)}{\left(2 n+0.5-\operatorname{Re} \alpha\left(M_{X}^{2}\right)\right)^{2}+\left(\operatorname{Im} \alpha\left(M_{X}^{2}\right)\right)^{2}}\right. \\
& \left.\quad+c \frac{f^{2}(t) M_{\text {Roper }} \Gamma_{\text {Roper }} / 2}{\left(M_{X}^{2}-M_{\text {Roper }}^{2}\right)^{2}+\left(\Gamma_{\text {Roper }} / 2\right)^{2}}\right),
\end{aligned}
$$

where $M_{\text {Roper }}=1440 \mathrm{MeV}, \Gamma_{\text {Roper }}=325 \mathrm{MeV}$, and $c$ is another normalization parameter. For illustration, we will take two different values of $c$; the resulting shapes of the double differential cross section are presented at Fig. 12.

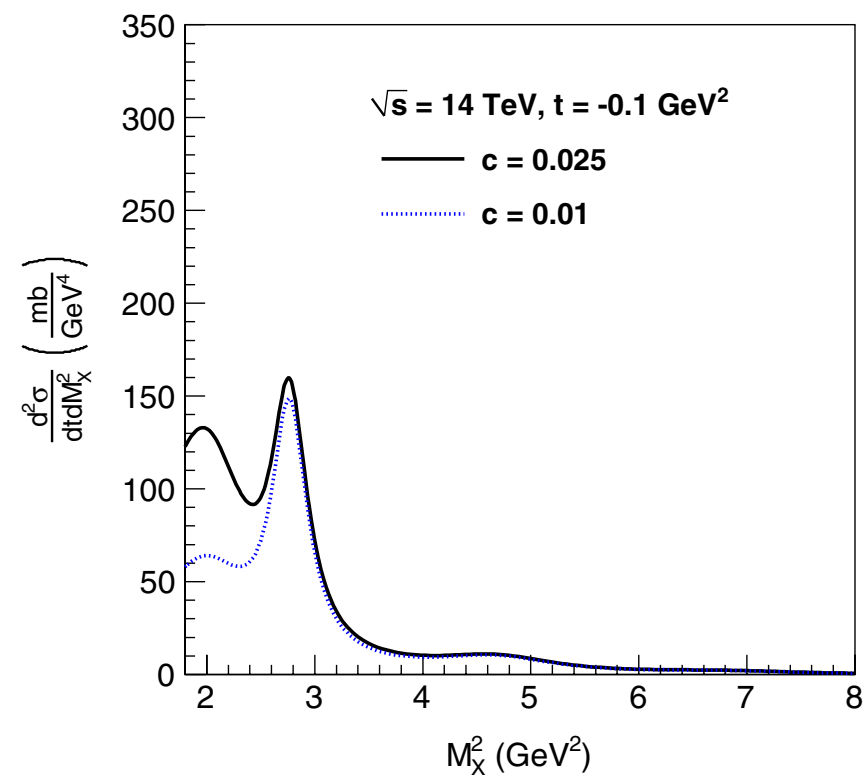

FIG. 12 (color online). Double differential cross section of SDD as a function of $M_{X}^{2}$ at fixed values of $s$ and $t$, with the Roper resonance added according to Eq. (A1).
[1] I. Ya. Pomeranchuk and E. L. Feinberg, Dokl. Akad. Nauk SSSR 93, 439 (1953) (in Russian); E. L. Feinberg and I. Ya. Pomeranchuk, Suppl. Nuovo Cim. 3, 652 (1956).

[2] M. L. Good and W.D. Walker, Phys. Rev. 120, 1857 (1960).

[3] N.P. Zotov and V.A. Tzarev, Usp. Fiz. Nauk 154, 207 (1988)[Soviet Physics Uspekhi 31, 119 (1988).

[4] R.D. Schamberger et al., Phys. Rev. Lett. 34, 1121 (1975).

[5] M. Albrow et al., Nucl. Phys. B108, 1 (1976); J.C.M. Armitage et al., Nucl. Phys. 194, 365 (1982).

[6] R. D. Schamberger et al., Phys. Rev. D 17, 1268 (1978).

[7] M. Albrow et al. USCMS Collaboration, JINST 4, P10001 (2009) [arXiv:0811.0120 [hep-ex]].

[8] K. Goulianos, Phys. Rep. 101, 169 (1983).

[9] D. P. Roy and R. G. Roberts, Nucl. Phys. B77, 240 (1974).

[10] G. A. Jaroszkiewicz and P. V. Landshoff, Phys. Rev. D 10, 170 (1974).

[11] A. Donnachie and P. V. Landshoff, Nucl. Phys. B244, 322 (1984).

[12] K. Goulianos and J. Montanha, Phys. Rev. D 59, 114017 (1999).

[13] S. Donnachie, G. Dosch, P. Landshoff, and O. Nachtmann, Pomeron Physics and QCD_; (Cambridge University Press, Cambridge, 2002); P. V. Landshoff, The Total Cross Section at the LHC, Lectures at the Copanello
Summer School, Calabria, 2007, [Acta Phys. Pol. B 39, 2063 (2008)].

[14] R. Fiore, L. Jenkovszky, R. Orava, E. Predazzi, A. Prokudin, and O. Selyugin, Int. J. Mod. Phys. A 24, 2551 (2009); arXiv:0812.0539 [hep-ph].

[15] R. Fiore et al., Eur. Phys. J. A 15, 505 (2002).

[16] R. Fiore et al., Phys. Rev. D 69, 014004 (2004).

[17] L. Jenkovszky, V. K. Magas, and E. Predazzi, Eur. Phys. J. A 12, 361 (2001).

[18] N. A. Amos et al., Phys. Lett. B 247, 127 (1990).

[19] F. Ravndal, Phys. Lett. B 37, 300 (1971).

[20] K. Goulianos, Phys. Lett. B 358, 379 (1995).

[21] P.D. G. Collins, An Introduction to Regge Theory and High Energy Physics (Cambridge University Press, Cambridge, 1977).

[22] C. Carlson and N.C. Mukhopadhyay, Phys. Rev. D 58, 094029 (1998).

[23] A. I. Bugrij et al., Fortschr. Phys. 21, 427 (1973).

[24] V. K. Magas, Yad. Fiz. 68, 106 (2005) [Phys. At. Nucl. 68, 104 (2005)]; arXiv:hep-ph/0404255; arXiv:hep-ph/ 0411335; Proc. Sci., DIFF2006 (2006) 051 [arXiv:hep$\mathrm{ph} / 0611119]$.

[25] Note that resonances on the proton trajectory appear with spins $J=1 / 2,5 / 2,9 / 2,13 / 2 \ldots$.

[26] In an alternative approach of Ref. [24], form factors enter with the same power for all the resonances on a given trajectory. The advantage of the models with increasing 
powers of the form factors is that the poorly known high spin resonances are strongly suppressed and thus do not affect the final results.

[27] R. Fiore, L.L. Jenkovszky, F. Paccanoni, and A. Prokudin, Phys. Rev. D 70, 054003 (2004); arXiv:hep-ph/0404021.

[28] C. Amsler et al. (Data Particle Group Collaboration), Phys. Lett. B 667, 1 (2008).

[29] A. Bodek et al., Phys. Rev. D 20, 1471 (1979).

[30] I. Niculescu, Ph.D. thesis, Hampton University, 1999.

[31] A. A. Arkhipov, arXiv:hep-ph/0012349v1.

[32] L.L. Jenkovszky, V. K. Magas, and O.E. Kuprash, in Proceedings of the International Workshop on Diffraction in High-Energy Physics (Diffraction 2010), Otranto, Lecce, Italy, 2010, (to be published).
[33] L. L. Jenkovszky, E.S. Martynov, and F. Paccanoni, in Proceedings of the "HADRONS-96" Conference, Novy Svet, Crimea, 1996.

[34] L. Jenkovszky, O. Kuprash, J. W. Lamsa, and Risto Orava (unpublished).

[35] B. E. Ansorge et al., Z. Phys. C 33, 175 (1986).

[36] G. J. Alner et al. (UA5 Collaboration), Phys. Rep. 154, 247 (1987).

[37] F. Abe et al. (CDF Collaboration), Phys. Rev. D 50, 5535 (1994).

[38] R. L. Cool, K. Goulianos, S. L. Segler, H. Sticker, and S. N. White (Fermilab E396), Phys. Rev. Lett. 47, 701 (1981).

[39] D. Bernard et al., Phys. Lett. B 186, 227 (1987).

[40] N. A. Amos et al. (E710 Collaboration), Phys. Lett. B 301, 313 (1993). 